\title{
The scalar spectrum of many-flavour $\mathrm{QCD} *$
}

\author{
Yasumichi Aoki ${ }^{a}$, Tatsumi Aoyama ${ }^{a}$, Masafumi Kurachi $^{a}$, Toshihide Maskawa $^{a}$, Kei-ichi Nagai $^{a}$, \\ Hiroshi $\mathrm{Ohki}^{a}$, Enrico Rinaldi ${ }^{a}$, 丹, Akihiro Shibata ${ }^{c}$, Koichi Yamawaki $^{a}$, Takeshi Yamazaki $^{a}$ \\ LatKMI collaboration \\ ${ }^{a}$ Kobayashi-Maskawa Institute for the Origin of Particles and the Universe (KMI), \\ Nagoya University, Nagoya, 464-8602, Japan \\ ${ }^{b} S U P A$ and School of Physics and Astronomy, University of Edinburgh, \\ Edinburgh, EH9 3JZ, UK \\ ${ }^{c}$ Computing Research Center, High Energy Accelerator Research Organization (KEK), \\ Tsukuba 305-0801, Japan
}

\begin{abstract}
The LatKMI collaboration is studying systematically the dynamical properties of $N_{f}=$ $4,8,12,16 \mathrm{SU}(3)$ gauge theories using lattice simulations with (HISQ) staggered fermions. Exploring the spectrum of many-flavour QCD, and its scaling near the chiral limit, is mandatory in order to establish if one of these models realises the Walking Technicolor scenario. Although lattice technologies to study the mesonic spectrum are well developed, scalar flavour-singlet states still require extra effort to be determined. In addition, gluonic observables usually require large-statistic simulations and powerful noise-reduction techniques. In the following, we present useful spectroscopic methods to investigate scalar glueballs and scalar flavour-singlet mesons, together with the current status of the scalar spectrum in $N_{f}=12$ QCD from the LatKMI collaboration.
\end{abstract}

Keywords: Lattice Gauge Theories, Walking Technicolour, Glueballs

\section{Introduction}

It has been known for more than 30 years that the electroweak symmetry breaking (EWSB) mechanism could be due to a new strongly-interacting sector at energies of $\mathcal{O}(\mathrm{TeV})$. Since then, this idea has evolved to become one of the most promising scenarios for physics beyond the Standard Model, the so-called Walking Technicolour with a large anomalous dimension $\gamma_{m} \sim 1$ and an approximate scale invariance ${ }^{1}$ (See also similar works ${ }^{2 / 4}$ without notion of anomalous dimension and scale invariance).

Recently, the discovery of a new particle of mass $\sim 125 \mathrm{GeV}$ at the Large Hadron Collider ${ }^{516}$ has attracted a lot of attention and it is now a pressing issue to determine its properties and its nature. The new particle's branching ratios are consistent with the ones of the Standard Model Higgs boson, but the very interesting possibility of

*Talk given at KMI-GCOE Workshop on Strong Coupling Gauge Theories in the LHC Perspective (SCGT 12), KMI, Nagoya University, December 4-7, 2012.

†Speaker. E-mail: e.rinaldi@sms.ed.ac.uk 
this particle being of composite nature, a techni-dilaton, 1 as predicted by Walking Technicolour models is also viable. ${ }^{78}$ The LHC experiments will be working very hard in the following years, trying to assess the validity of their analysis for the different decay channels of the new particle. At the same time, the effort of the theory community will go in a similar direction; new increasingly precise lattice simulations might help addressing the question whether or not a more fundamental strongly-interacting gauge sector is responsible for the origin of mass (a review of recent lattice studies can be found in Refs. 9/10).

The LatKMI collaboration is focusing on a systematic study of SU(3) gauge theories with different numbers $N_{f}$ of fundamental massless fermions using lattice Monte Carlo simulations (see Refs. 11 13 for recent updates). The main purpose of such on-going project is to find a near-conformal theory with a large anomalous dimension $\gamma_{m} \sim 1$ by investigating the infrared dynamics of the theory which is in the non-perturbative domain. If such a theory will be found, the important question will be to identify the composite scalar state, techni-dilaton, mimicking the role of the Higgs boson of the Standard Model and to study its mass as it arises from a purely non-perturbative dynamics.

The study of scalar single-particle resonances on the lattice is complicated due to the poor quality of the signal-to-noise ratio in Monte Carlo simulation. Even in state-of-the-art lattice QCD calculations, where an extreme degree of accuracy has been reached in recent years for many physical observables, the spectrum of scalar mesons is still an open issue. Moreover, scalar excitations of gluonic degrees of freedom (glueballs) introduce an additional factor of complexity since they can mix with fermionic states: a careful study is necessary to distinguish the nature of states in the scalar channel (for an up to date review see Ref. 14). In the following, we describe the status of LatKMI investigation of the scalar spectrum from lattice simulations of $N_{f}=12 \mathrm{QCD}$. The data shown in the following are preliminary.

\section{Lattice simulations}

The lattice gauge configurations for the $\mathrm{SU}(3)$ theory with $N_{f}=12$ fundamental flavours are generated by the HMC algorithm using a tree-level Symanzik gauge action and the HISQ (Highly Improved Staggered Quark ${ }^{15}$ ) action for the dynamical fermions. The flavour symmetry breaking of this action (measured as taste splitting) is highly suppressed in $\mathrm{QCD}^{16}$ and it is almost negligible for the theory we investigate. At the couplings used in this study, the masses of bound states in the same taste multiplet can not be distinguished from one another as shown in Fig. 1 , More details on the calculated mesonic spectrum and decay constants can be found in Ref. 11.

At fixed lattice spacing defined by the bare coupling constant $\beta=6 / g^{2}=$ 4.0, we simulate two physical volumes $V=(a L)^{3}$ with $L=18,24$ and aspect ratio $T / L=4 / 3$. On the smaller volume we investigate the scalar spectrum at five different bare quark masses $0.06<a m_{f}<0.16$; the bare mass changes by almost 


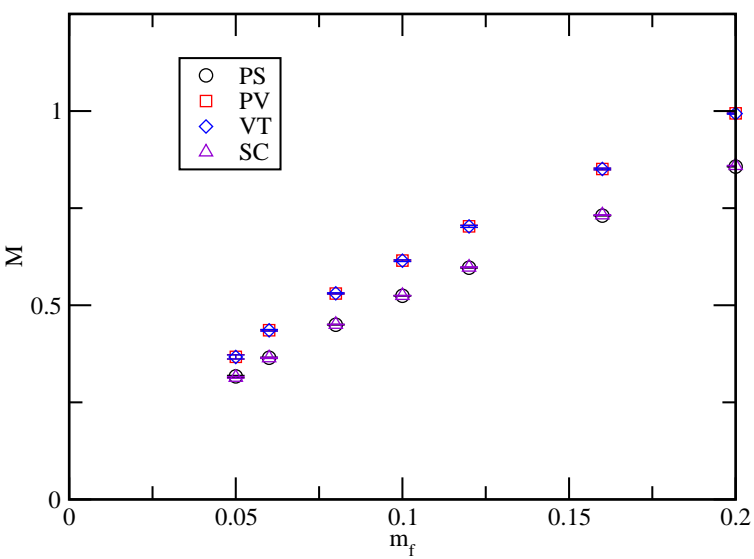

Fig. 1. An example of the small taste splitting in the spectrum of $N_{f}=12$ QCD with HISQ fermions at $\beta=4.0$. The figure is taken from Ref. [11, $\mathrm{M}$ and $m_{f}$ are the meson mass and the bare quark mass respectively, both in units of the common fixed lattice spacing $a$. PS and SC refer to pseudoscalar mesons coming from operators with different internal taste structure, whereas PV and VT refer to vector mesons.

a factor of 3 and this allows us to identify a possible quark mass dependence in the measured observables. Another important feature of our simulation is the large number of Monte Carlo trajectories obtained from uninterrupted Markov chains after thermalization. For any set of parameters explored, we collect more than 15000 trajectories (with MD step set to unity), which is necessary to contrast the rapid degradation of signal to noise ratio of the scalar correlators. A summary of the simulated parameters is found in Tab. 1.

Table 1. Parameters of lattice simulations for $N_{f}=12 \mathrm{QCD}$ at fixed $\beta=4.0 . N_{\text {traj }}$ is the number of Monte Carlo trajectories after thermalisation, $N_{\text {cfgs }}$ is the number of saved gauge configurations. For different ensembles, configurations are saved every 2 or 5 trajectories.

\begin{tabular}{llll}
\hline$L \times T$ & $a m_{f}$ & $N_{\text {traj }}$ & $N_{\text {cfgs }}$ \\
\hline $18 \times 24$ & 0.06 & 15445 & 3090 \\
$18 \times 24$ & 0.08 & 22200 & 4440 \\
$18 \times 24$ & 0.10 & 15300 & 3060 \\
$18 \times 24$ & 0.12 & 21500 & 4300 \\
$18 \times 24$ & 0.16 & 18450 & 3690 \\
$24 \times 32$ & 0.06 & 28000 & 14000 \\
$24 \times 32$ & 0.08 & 19280 & 9640 \\
$24 \times 32$ & 0.10 & 18960 & 9480 \\
\hline
\end{tabular}




\section{Glueball measurement}

It has been pointed out ${ }^{17}$ that signs of infrared conformality can be found by comparing the mesonic spectrum with the gluonic one. Such a comparison was done in the $\mathrm{SU}(2)$ gauge theory with two flavours of adjoint fermions ${ }^{18}$ and the results supported the hypothesis of an infrared fixed point being responsible for the dynamics of the theory on the critical massless surface.

Addressing a measurement of the glueball spectrum is intrinsically difficult due to the poor signal over noise ratio in gluonic correlators. The theory behind the measurement of glueball masses in lattice spectroscopy has been known for a long time ${ }^{19}$ Recently, a powerful framework to measure gluonic bound states has been developed ${ }^{\sqrt{20}}$ that improves upon previous results. The method employs a large number of different operators built from gauge-invariant combinations of gauge links in such a way that a robust basis for a variational ansatz can be created. The variational approach, with such a large basis, has been shown to produce excellent results for different gauge theories.20 22 In the following we briefly review the technology involved in such a variational method and we show the preliminary results obtained by the LatKMI collaboration using the gauge configurations in Tab. 1, This is the first time that glueball states are investigated in many-flavour SU(3) gauge theory. Eigenstates of the lattice Hamiltonian fall into irreducible representations of the symmetry group characterising the four-dimensional lattice system. In particular, there are 20 symmetry channels for glueballs on a three-dimensional spatial lattice, corresponding to the 5 irreducible representations of the cubic group and including both parity and charge quantum numbers. In the following, we focus on the scalar $0^{++}$channel that is expected to feature the lightest state. On each spatial point of the lattice we build gauge-invariant interpolating operators $\mathcal{O}_{\alpha}(x, t)$ with well-defined rotational quantum number by using a prescribed linear combination of traced spatial Wilson loops. Zero-momentum operators consist in an average over the whole volume $V$. By using differently shaped Wilson loops we construct 32 different basis operators for the scalar glueball (each of these operators come in different blocking sizes and they are all included in the variational basis).

Let us now give an example of a typical glueball measurement. For every configuration we measure a matrix of correlation functions, whose elements are

$$
\tilde{C}_{\alpha \beta}(t)=\sum_{\tau}\left\langle 0\left|\mathcal{O}_{\alpha}^{\dagger}(t+\tau) \mathcal{O}_{\beta}(\tau)\right| 0\right\rangle .
$$

By solving the generalised eigenvalue problem for the matrix above, optimal operators (i.e. those that create almost pure states $|i\rangle$ ) can be found that are a linear sum of the basis vectors

$$
\tilde{\mathcal{O}}_{i}(t)=\sum_{\alpha} v_{\alpha}^{i} \mathcal{O}_{\alpha}(t) ; \quad \tilde{\mathcal{O}}_{i}(t)|0\rangle \approx|i\rangle,
$$

where $v_{\alpha}^{i}$ are the components of the $i^{\text {th }}$ eigenvector. Different eigenvectors $v^{i}$ correspond to different states. The mass $m_{i}$ of the $i^{\text {th }}$ state is then extracted by fitting 
correlators of optimal operators using

$$
\bar{C}_{i i}(t)=\left|c_{i}\right|^{2}\left(e^{-m_{i} t}+e^{-m_{i}(T-t)}\right),
$$

where $T$ is the length of the lattice in the time direction and the functional form is a consequence of the usual exponential decay in a lattice with periodic boundary condition in the time direction.

In general, glueball correlators are very noisy and this limits the usefulness of numerical correlators to short time separations. However, although Eq. (3) is only valid at large $t$, if the overlap with an Hamiltonian state is almost perfect, it is possible to extract a reliable value for the mass at short time separation, since the decay is largely dominated by a single state. For this to be true, a careful construction of the variational basis is paramount. Whether the state created by an optimal operator $\tilde{\mathcal{O}}_{i}(t)$ is a good approximation of the Hamiltonian eigenstates can be checked by looking at the value of the overlap $\left|c_{i}\right|^{2}$ : the closer this number is to one, the better is the variational calculation.

We measure the correlators of scalar gluonic operators on the configurations of Tab. 1. The measurements are taken for every configurations and statistical errors are estimated using jack-knife. We check for autocorrelations by changing the size of the jack-knife bins.

For each set of simulated parameters, we perform the variational analysis described above and we obtain the correlator corresponding to the optimal operator that projects on the ground state $(i=1)$. In some cases, the correlator for the first excited state $(i=2)$ is studied as well in order to estimate the higher states contamination in the ground state estimate. We calculate the effective mass and we look for a plateaux where Eq. (3) can be applied. An example of the scalar ground state correlator and its effective mass is shown in Fig. 2 ,

The high number of analysed configurations is very helpful when determining the effective mass plateaux. For example Fig. 3] shows how the effective mass changes when the statistic is increased.

\section{Flavour-singlet scalar meson}

In the staggered discretization, fermionic fields defined on the lattice sites have an additional taste structure. The scalar $0^{+}$fermionic interpolating operator that we used in the construction of our propagators is the simplest one and has a $(1 \times 1)$ spin-taste content. As is generally the case, this operator couples also to a negative parity $0^{-}$state whose contribution must be accounted for in the analysis of the full connected correlator $C(t)$. However, for the scalar flavour-singlet case (referred to as $\sigma$ in the following), we need to compute disconnected diagrams and the vacuum expectation value of the mesonic operator.

Measuring disconnected contributions to mesonic observables is very challenging and ad-hoc noise reduction techniques have been developed and used successfully in lattice QCD (LQCD). We used the stochastic source method ${ }^{23 \mid 24}$ with Gaussian 


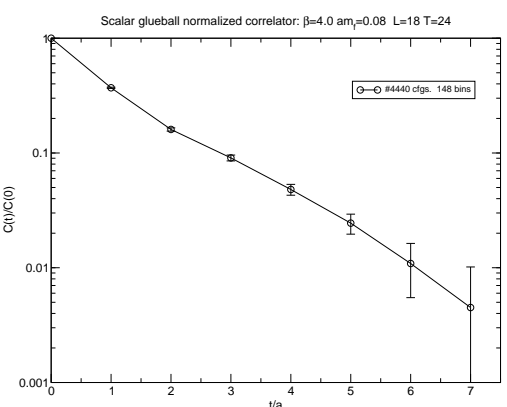

(a)

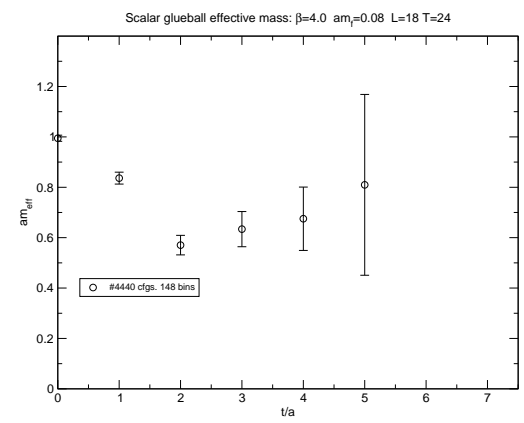

(b)

Fig. 2. Analysis of 4440 configurations for $\beta=4.0$ and $a m_{f}=0.08$ on the smaller volume. (a) The time dependence of the normalized scalar correlator in logarithmic scale; the linear behaviour corresponds to the propagation of a single state (b) The effective mass of the corresponding correlator; the signal is lost after 6 timeslices, but the plateaux is already present at short temporal distances.

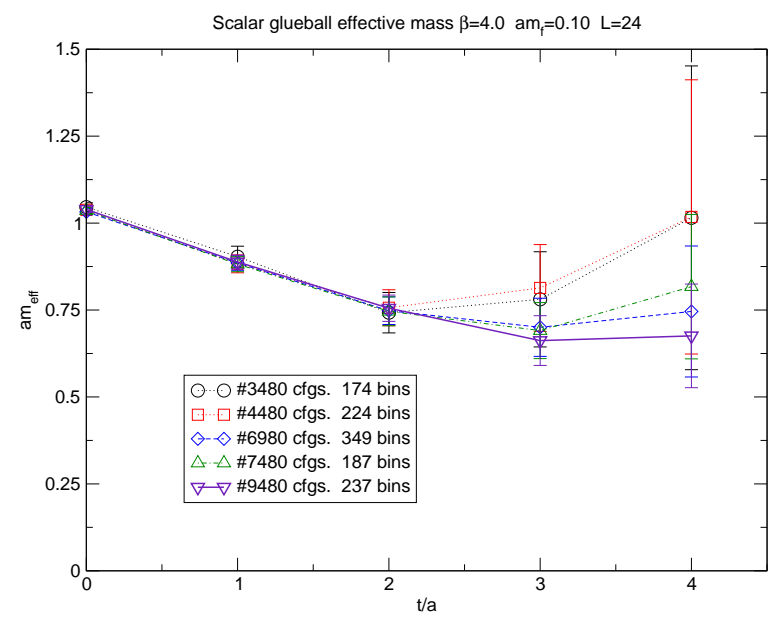

Fig. 3. An example of the scalar effective mass at $\beta=4.0$ and $a m_{f}=0.10$ on the larger volume $L \times T=24 \times 32$ as the number of configuration is increased. A stable plateaux becomes more and more visible as the statistics increases.

noise and local source and sink insertions. The number of noise vectors for each configuration has been chosen to the minimal value that yields the asymptotic value of the disconnected correlator $D(t)$ on the available statistics.

Thanks to the relative high number of configurations analysed at the lightest bare fermion mass (cfr. Tab. 1), we are able to extract a clean signal for the disconnected correlator $D(t)$ at large temporal distances and including the vacuum expectation value subtraction, as it is shown in Fig. 4 (a). The scalar singlet correlator $C_{\sigma}(t)$ is extracted from combinations of $C(t)$ and $D(t)$ that properly account for the effects 


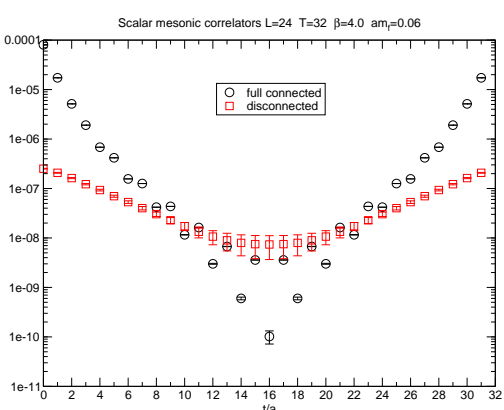

(a)

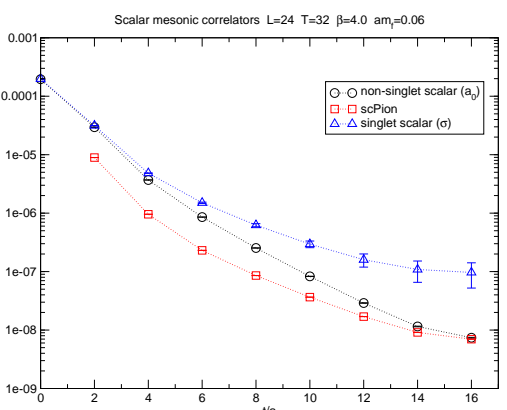

(b)

Fig. 4. Results for the analysis of scalar mesonic interpolating operators (a) The full connected and disconnected correlators where the vev as been subtracted in the latter. The connected correlator still includes the parity partner contributions. (b) The correlators of the different channels extracted from $C(t)$, where disconnected terms have been included for the singlet case only.

of the parity partner meson and of the vacuum fluctuations:

$$
\begin{aligned}
\tilde{C}(2 t) & =2 C(2 t)+C(2 t+1)+C(2 t-1), \\
\tilde{D}(2 t) & =2 D(2 t)+D(2 t+1)+D(2 t-1), \\
C_{\sigma}(2 t) & =-\tilde{C}(2 t)+3 \tilde{D}(2 t) .
\end{aligned}
$$

The factor of 3 in the last equation comes from the number of actual fermions in the simulation ( 3 staggered fermions $\times 4$ tastes $=12$ flavours). From the full connected correlator, the non-singlet $\left(a_{0}\right)$ and the pseudoscalar (scPion) states can be isolated as well. The three different channels are compared in Fig. 目(b) and the results strongly indicates that the $\sigma$ meson mass is smaller than the pseudoscalar one. Moreover, although the scalar effective mass plateaux appears only at very large temporal separation and the statistical error are large, the corresponding mass is compatible with the one obtained using only purely gluonic operators. The comparison between the gluonic scalar spectrum and the fermionic scalar singlet is reported in Fig. [5.

\section{Conclusions}

We performed a study of the scalar glueball and scalar flavour-singlet fermionic state. Advanced lattice spectroscopy techniques that have proven successful in LQCD studies have been applied for the first time to explore the scalar spectrum of many-flavour QCD.

The results shown in the previous sections clearly show that the variational method used to extract masses of gluonic bound states is very efficient and allowed us to study the qualitative quark mass dependence of scalar glueballs. Although the results are preliminary and the statistical errors of the order $\sim 10 \%$, it is very encouraging that a signal for an effective mass plateaux could be obtained even for relatively small time separation. As for the scalar fermionic correlator including the 
disconnected term, although an effective mass plateaux is observed at somewhat larger time due to the lack of improved operators, it is remarkable that the effective mass is consistent with the mass obtained from the gluonic operators.

The most striking feature of the measured scalar spectrum is the appearance of a state lighter than the pion for low bare quark masses as it is shown in Fig. 5 . Such a state appears both in gluonic and fermionic correlators, indicating that mixing between gluons and fermions plays a relevant role. A future variational analysis including both kind of operators described in the previous sections will help disentangle mixed contributions. Moreover, a careful finite size study is under completion and possible systematic effects of the lattice regularization are being investigated. Despite it being studied by several groups using different approaches, $N_{f}=12$ QCD has not yet been identified as an infrared-conformal or near-conformal theory. The majority of studies suggest the presence of an infrared fixed point with a small anomalous dimension. If this turns out to be the case, such a theory would not be a viable candidate for a phenomenologically interesting Walking Technicolour model. A walking theory, which may be realised near the edge of the conformal window, should have its property influenced by the near-conformal theory. The light scalar state observed for $N_{f}=12$ in this study is a promising signal for the search of a successful walking theory, where the existence of a light scalar is now required by LHC experiment. A possible future direction is to look at more viable candidates for Walking Technicolour models; for example, it will be interesting to investigate the scalar spectrum of the $N_{f}=8 \mathrm{SU}(3)$ theory where, if the theory has a walking regime, the scalar state could be identified with the pseudo-NG boson coming from the breaking of conformal symmetry.

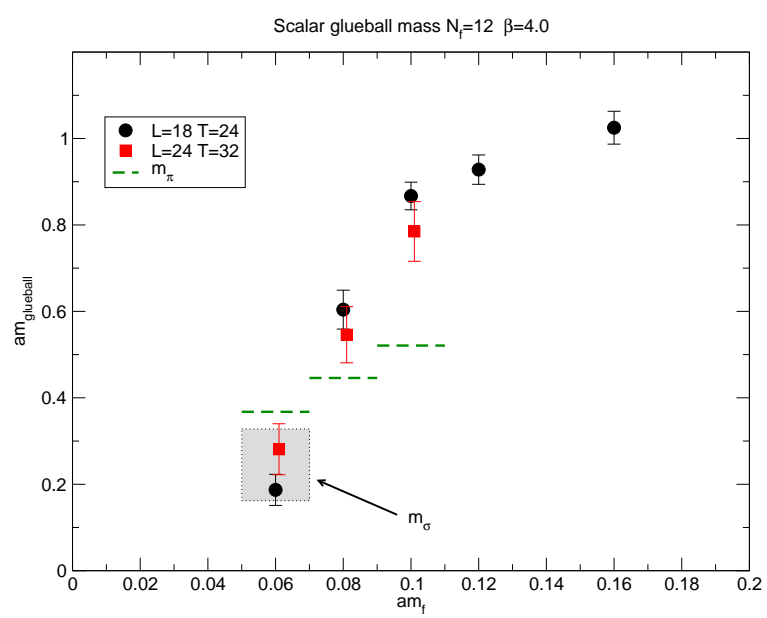

Fig. 5. Summary of the fitted scalar glueball masses for $N_{f}=12 \mathrm{QCD}$ at $\beta=4.0$ and for several bare fermion masses. Some values of the pion mass are shown for comparison. The point on different volumes have been slightly displaced for clarity. The grey box indicates the location of the scalar flavour-singlet mass $m_{\sigma}$ on the ensemble with the lightest bare quark mass. 


\section{Acknowledgments}

Numerical simulation has been carried out on the supercomputer system $\varphi$ at KMI in Nagoya university, and on the computer facilities of the Research Institute for Information Technology at Kyushu University.

This work is supported by the JSPS Grant-in-Aid for Scientific Research (S) No.22224003, (C) No.23540300 (K.Y.) and (C) No.21540289 (Y.A.), and also by Grants-in-Aid of the Japanese Ministry for Scientific Research on Innovative Areas No. 23105708 (T.Y.). E.R. is currently supported by a SUPA Prize Studentship and was supported by a FY2012 JSPS Postdoctoral Fellowship for Foreign Researchers (short-term), ID PE12518.

\section{References}

1. K. Yamawaki, M. Bando and K.-i. Matumoto, Phys.Rev.Lett. 56, p. 1335 (1986).

2. B. Holdom, Phys.Lett. B150, p. 301 (1985).

3. T. W. Appelquist, D. Karabali and L. Wijewardhana, Phys.Rev.Lett. 57, p. 957 (1986).

4. T. Akiba and T. Yanagida, Phys.Lett. B169, p. 432 (1986).

5. CMS, Phys.Lett. B716, 30 (2012).

6. ATLAS, Phys.Lett. B716, 1 (2012).

7. S. Matsuzaki and K. Yamawaki, Phys.Rev. D86, p. 035025 (2012).

8. S. Matsuzaki and K. Yamawaki, Phys.Rev. D86, p. 115004 (2012).

9. E. T. Neil, $P o S$ LATTICE2011, p. 009 (2011).

10. J. Giedt, PoS LATTICE2012, p. 006 (2012).

11. Y. Aoki, T. Aoyama, M. Kurachi, T. Maskawa, K.-i. Nagai, H. Ohki, A. Shibata, K. Yamawaki and T. Yamazaki, Phys.Rev. D86, p. 054506 (2012).

12. Y. Aoki, T. Aoyama, M. Kurachi, T. Maskawa, K.-i. Nagai, H. Ohki, A. Shibata, K. Yamawaki and T. Yamazaki, PoS LATTICE2012, p. 035 (2012).

13. Y. Aoki, T. Aoyama, M. Kurachi, T. Maskawa, K.-i. Nagai, H. Ohki, A. Shibata, K. Yamawaki and T. Yamazaki, PoS LATTICE2012, p. 029 (2012).

14. W. Ochs, arxiv:1301.5183 (2013).

15. E. Follana et al., Phys.Rev. D75, p. 054502 (2007).

16. A. Bazavov, T. Bhattacharya, M. Cheng, C. DeTar, H. Ding et al., Phys.Rev. D85, p. $054503(2012)$.

17. L. Del Debbio, B. Lucini, A. Patella, C. Pica and A. Rago, Phys.Rev. D80, p. 074507 (2009).

18. L. Del Debbio, B. Lucini, A. Patella, C. Pica and A. Rago, Phys.Rev. D82, p. 014510 (2010).

19. B. Berg and A. Billoire, Nucl. Phys. B221, p. 109 (1983).

20. B. Lucini, A. Rago and E. Rinaldi, JHEP 1008, p. 119 (2010).

21. L. Del Debbio, A. Hart and E. Rinaldi, JHEP 1207, p. 178 (2012).

22. E. Gregory, A. Irving, B. Lucini, C. McNeile, A. Rago et al., JHEP 1210, p. 170 (2012).

23. L. Venkataraman and G. Kilcup, Phys.Rev.D (1997).

24. E. B. Gregory, A. C. Irving, C. M. Richards and C. McNeile, Phys.Rev. D77, p. 065019 (2008). 